\title{
Communication and Computation in Buildings: A Short Introduction and Overview
}

\author{
Dietmar Dietrich, Senior Member, IEEE, Dietmar Bruckner, Senior Member, IEEE, Gerhard Zucker, Member, IEEE, \\ and Peter Palensky, Senior Member, IEEE
}

\begin{abstract}
Building automation (BA) and smart homes (SHs) have traditionally not been a unified field but varied by their origins, legal foundations, different applications, different goals, and national funding programs for basic research. Only within the last years that an international common focus appeared. The following overview gives not only an introduction into the topic of $\mathrm{BA}$ but also the distinction to other areas of automation, in which networks of the field level (the sensor and actuator level) play an important role. Finally, the scientific challenges will be mentioned. SHs are referred to when the differences to BA have to be explicitly stressed. This paper is an introduction for the special IEEE Transactions on INDUSTRIAL Electronics section on BA and shall introduce the reader to this new topic. BA not only has a huge economic potential but also is of significant academic interest today.
\end{abstract}

Index Terms-Ambient assisted living, building automation (BA), building control, building management, communication networks, embedded systems, energy management, fieldbus systems, horizontal integration, industries, layered architecture, smart homes (SHs).

\section{Domain}

B UILDING AUTOMATION (BA) is concerned with control and communication networks in buildings; the systems consist of sensors, actuators, and communication and processing units. The devices are mostly interconnected using dedicated fieldbus systems, which-in opposition to industrial fieldbuses-require more flexibility, management, and interoperability and therefore have more layers of the International Organization for Standardization (ISO)/Open System Interconnection (OSI) model implemented. The term smart home (SH) refers to a communication network that combines BA components with other communication systems and informationsharing components in private homes (home cinema equipment, computer networks, security, e-mail, calendar, file-sharing services, etc.).

Manuscript received December 31, 2008; revised March 17, 2010; accepted March 29, 2010. Date of publication August 8, 2010; date of current version October 13,2010.

D. Dietrich and D. Bruckner are with the Institute of Computer Technology, Vienna University of Technology, 1040 Vienna, Austria (e-mail: dietrich@ ieee.org).

G. Zucker was with Vienna University of Technology, 1040 Vienna, Austria. $\mathrm{He}$ is now with the Austrian Institute of Technology, 1210 Vienna, Austria.

P. Palensky is with the Austrian Institute of Technology, 1210 Vienna, Austria.

Digital Object Identifier 10.1109/TIE.2010.2046570

\section{INDUSTRIES AND COSTS}

Up to the 1990s, the expressions BA and SHs were absent from encyclopedias like Webster's [1] or the German Brockhaus [2]. Many people, including engineers and scientists, still have problems with these expressions. A query in the English edition of Wikipedia [3] lists classic technologies which are still found in the European market (particularly Germany and Scandinavia) like the $20 \mathrm{~mA}$ or RS 232 interface. The German edition of Wikipedia [4] lists BA as part of facility management but contains more recent technologies. Mainly, the three established fieldbus systems (BACNet, LonWorks, and KNX; see Section III) of the BA standards in the European Committee for Standardization (CEN) and ISO are mentioned (the English site lists 21 bus systems without any further explanations). In the following, we will give our view on communication in BA, also based on historical developments.

Although not comparable to classical encyclopedias, Wikipedia shows one important fact: de facto definitions and descriptions of topics that are otherwise hard to grasp. The notions of BA and SH differ among countries, particularly in academics. In the European understanding associates a long list of functions and technologies with BA. These can only be implemented cost efficiently when using a fieldbus system.

In America, fieldbus systems in BA or even $\mathrm{SH}$ were, in practice, less important. For example, if one is looking for components for $\mathrm{SH}$ in the American market, one finds Internet components, alarm systems, and components for brown goods (consumer electronics) and white goods (household appliances) with no or limited network management capabilities-or with a touch of do-it-yourself (e.g., the X10 protocol ${ }^{1}$ ). European markets, on the other hand, exclude components for brown and white goods from the BA industry.

It is also remarkable that, although BACNet and LonWorks [5] were developed in the U.S., BA and SH have not been implemented too much due to economical reasons in the American area, while the topic itself plays more and more a key role, particularly when being merged with the topic of saving energy. Only in the last few years that fieldbus technology became an economically important factor-which can be observed with the sales figures of Echelon or BACNet nodes.

Universities in Vienna, Dresden, Munich, Zurich, Aveiro, Sofia, Chatou, Heilbronn, Lisbon, or Dortmund (particularly the Faculties of Electrical Engineering, Computer Technology,

\footnotetext{
${ }^{1} \mathrm{X} 10$, however, cannot be compared with networks like BACNet, LonWorks, etc., regarding its functional range.
} 
and Computer Science) are cooperating in the field of BA [6]. In the non-European countries, it does not look so well (if we do not consider energy management), and the following questions arise: Why the non-European industry does not see the potentials of this market in the same way like the Europeans? Where do the differences come from? Worldwide, there is no doubt that efficient energy saving is only possible with modern BA based on networking in all levels of abstraction.

To get an answer, it is necessary to go far back in history. In the 15th and 16th centuries, the different industries and trades ("Gewerke" and "Zünfte," old terms for what we call "industries" [7] today) in Europe formed and became strong powers in their societies. Even today, these industries are still deeply rooted in traditions and have strictly divided markets. The different industries had nothing to do with each other and were and still are not used to work together, may it be in building sites or in contributing to standardization organizations. All of them developed their own lobbies to get special rights, which helped to build up strong markets in their areas. Today, we see this fundamental thinking in the standardization organization where different industries form different groups, which have nothing to do with each other. In western Europe, electric installations, heating, ventilation, and plumbing all have their representative communities, which do not communicate sufficiently, and if a building is under construction, the different industries do not cooperate but work independently. The way of thinking is focused on working independently, also in standardization organizations [8]: For BA, it is much harder to accept that, up to today, most of the official calls for bids are based on this fundamentally divided market. How is it possible to get an offer which is based on networked components that include all industries-which would be the prerequisite for integrating all their functions as discussed later? For the organization units, the question is always as follows: Who are the accredited experts that are able to write a report of such a network-based building? An official education program does not exist yet. Experts, defined by fieldbus user organization like LonWorks, the so-called "integrators," are not accepted officially yet. In the opinion of the authors, ${ }^{2}$ too many interests work against each other and do not pull on the same string. Particularly in Europe, it is of highest priority to establish the profession of an integrator to strengthen the cooperation between the various industries and leave the responsibility for networking to one person. Due to historical reasons, this integration works much better in the U.S.

We now take a look at the fundamental problem of networked systems bridging components of various industries. In the history of science, computer scientists were the first to deal with networking; thus, they are the experts in this field. However, who can be considered an expert in the case of sensors that are integrated into a chimney and are networked with sensors, actuators, and controllers for heating and lighting systems over a common bus system? Who is able to standardize functions and scenarios that are provided by different industries? To be a computer engineer is not enough. The experts of the different

${ }^{2}$ D. Dietrich is a convenor of CEN TC 247 WG4 and a delegate of CEN TC 247 where the fieldbus systems are defined for CEN and proposed for ISO.

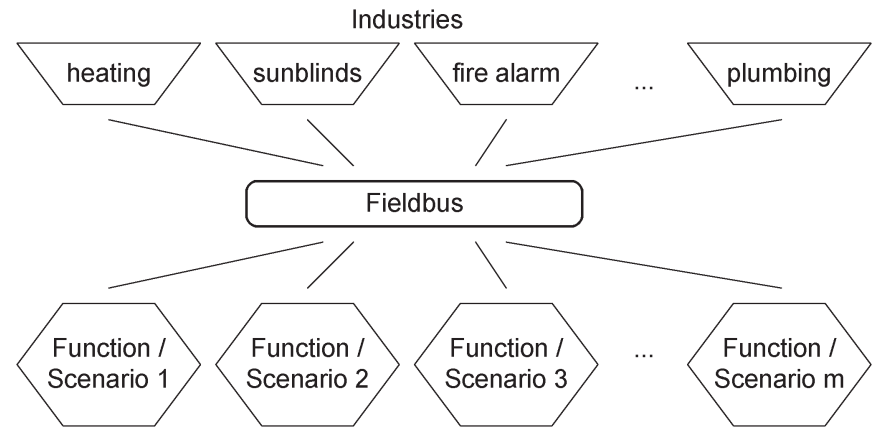

Fig. 1. Merging of various industries and subdivisions in different functions and scenarios.

industries have to sit together with computer engineers and find common standards. ${ }^{3}$ That is tough because of all the definitions of the different industries which developed independently over the centuries. Now, all these things must be harmonized and reconsidered. It is not surprising that efforts to change the situation have already taken more than 15 years. There are not only economic interests but also legal differences and social challenges for which we have to find balanced answers, and people who studied special professions, but were not interested in electronic systems, now have to deal with topics like communication protocol (see, e.g., [9] and [10]), networking over fieldbus systems [11], [12], and safety and security [13]. Creating the profession of the "integrator" was a reasonable development - only the legal accreditation is still a problem.

Fig. 1 should explain the difficult process. First, the various industries acted independently; today, they must cooperate and have to define and merge the many different functions and scenarios for BA and $\mathrm{SH}$. The fieldbus is the connecting link from which new challenges emerge, because most of the functions and scenarios need the technologies of other industries and cannot be developed independently.

Unfortunately, a special professional group had a negative impact on SH and the networked BA: the architects. They define themselves as designers and more and more as artists, to whom automation is not relevant. If possible, the "passive" building (without automation) should satisfy all demands. Automation is a "necessary evil" which should be included as little as possible. BA has very little to contribute to art. Even worse: electronics contain risks and who is to pay for it? However, architects had to learn that building owners think differently. They want the building to be profitable, which is possible by saving energy, reducing staff costs, and increased comfort to attract clients. Building owners of big buildings already think in such a differentiated way, and so, most of the huge buildings in Europe contain fieldbus systems as the basis of BA.

The situation outside of Europe is not so clear, since not much information is available. However, it is possible to generalize the most important aspects: In the U.S., Australia, etc., fortunately, industries never played such an important role and never had such influential lobbies working for the development of buildings. The energy and the personnel costs are lower

\footnotetext{
${ }^{3}$ Organizations like ASHRAE, LonWorks, or KNX are not accredited standardization organizations neither in Europe nor worldwide like ISO or International Electrotechnical Commission.
} 


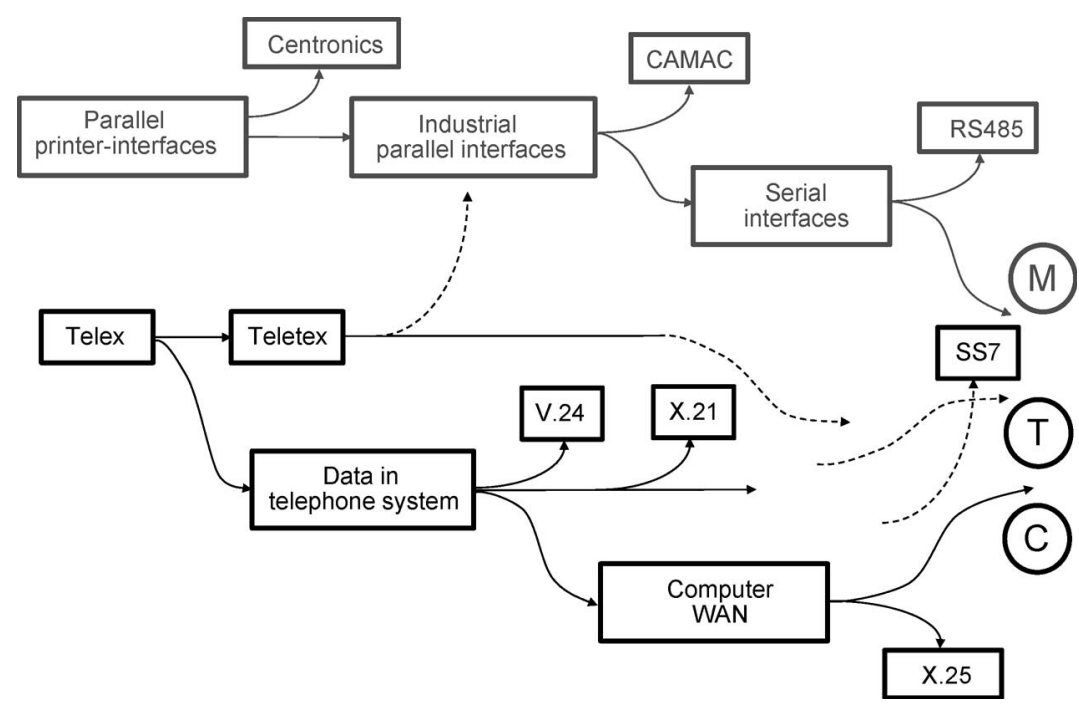

Fig. 2. Strict separation between the three areas, namely, measuring, telecommunication, and computer technology [16]. M: Measurement and Electrical Engineering. T: Telecommunication. C: Computer Technology.

in such countries, and comfort for the clients can already be achieved by classic technologies if the personnel costs are low enough.

We take a look at science and research in Section III where we explain the history of fieldbus systems. However, first, we look at publications in IEEE, which present a reasonable picture of those times. In the area of journals, it was-and still is - hard to find anything about this topic, because research work relies on funding. Outside Europe, in many countries, nobody saw the necessity to spend money on research, which is the reason for most experts to work in Europe and publish in national journals so far, where they reach their audience. First, international activities can be found in conferences like FeT 1995 (the current conference Web site can be found at [14]), which later joined the International Federation of Automatic Control. IEEE had nothing to offer in this topic, only later, for example in IEEE Workshop on Factory Communication Systems (the current conference Web site is located at [15]). Up to this day, most of the IEEE conferences, which deal with the topics of BA or even $\mathrm{SH}$, focus their interests more on the direction of real-time systems.

On the basis of this explanation, it is understandable that, about three years ago, the IEEE/IES founded the Technical Committee on Building Automation, Control and Management [6] in order to change the situation internationally. The huge market worldwide of BA and the high amount of energy that we can save this way are near at hand. Governments in most countries also recently recognized that and established partly substantial funding, e.g., in California. Finally, the scientific challenges are increasing dramatically, a topic which will be explained in Section V.

\section{Fieldbus Systems-History}

In [16], one view of the history of fieldbus systems is covered. In the area of automation, an existing printer interface (e.g., Centronics) from desktop computing in office areas was used to solve simple measurement tasks (Fig. 2). On the basis of such uncomplicated demands, various interfaces and bus systems are developed in industry and scientific laboratories where the influence of the telecommunication area was not really important. Only special interfaces were taken over-for example, RS 232 or, later, RS 485 (Fig. 2).

It was surprising for experts that telecommunication engineers did not understand that signal transmission could not be the whole story of communication systems. It completely slipped their mind to offer an answer for the computer scientists' question on how to connect their huge computers among the different towns over telecommunication lines. Finally, scientists from areas like computer science, mathematics, and computer technology defined the OSI model within the ISO but not in the Comité Consultatif International Téléphonique et Télégraphique, which would have been much more logical. They defined a model for protocols with various abstract levels as a template for protocol developers.

This model has become the basis for lots of protocols, not only the telecommunication area but also for automation systems (Fig. 3) like the Manufacturing Automation Protocol (MAP). MAP, developed by companies like General Motors, IBM, and many other American companies, was a typically overloaded protocol, very flexible, and with a lot of functions (all seven layers were included), but also with all disadvantages of such a powerful system: high maintenance costs, expensive to integrate, etc. The next generation was MiniMAP with only three layers but still too expensive for the market. The breakthrough was in contrast to this, the Standard MIL 1553 for the avionic area. It was also the basis for the first fly-bywire plane, the European Airbus A320. From this time on, one bus after the other was developed, the BITbus, CAN, Profibus, Interbus, and many others for various applications. Most of them were developed and standardized in Europe for the automotive and industrial automation market. Typical applications were assembly lines, filling lines, timber industry, etc. It was also the beginning of an international clash of national interests, with each country trying to assert its own standards. Therefore, for the first time, the European standardization organization standardized three fieldbus systems (Profibus, WorldFIP, and P-NET) from three different countries (Germany, France, and 


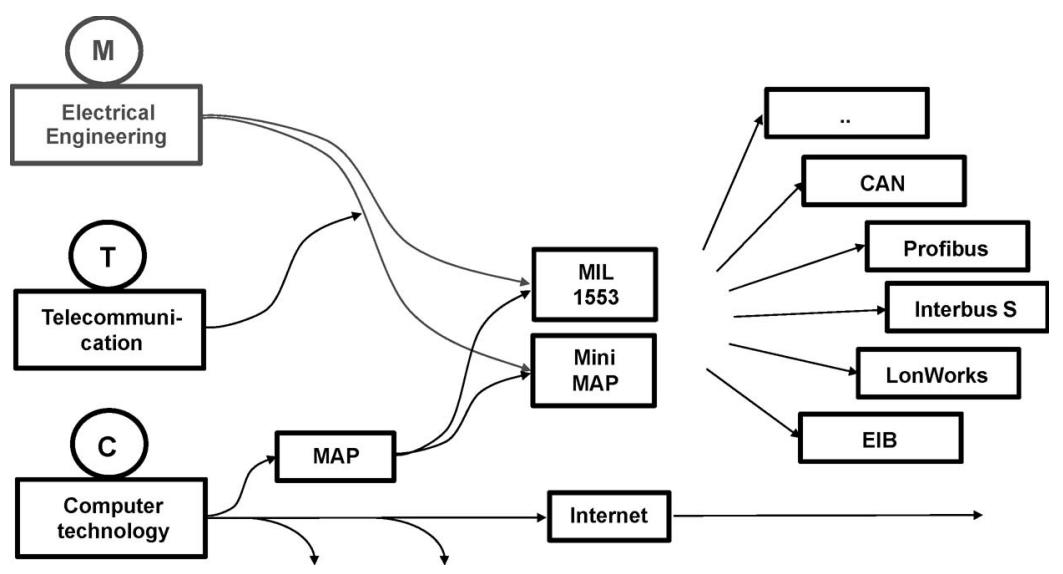

Fig. 3. Origins of fieldbus systems.

TABLE I

INDUSTRIES IN AUSTRIAN BUILDING AUTOMATION

\begin{tabular}{ll}
\hline \hline Heating technology & Telephone systems and EDP networks \\
Ventilation technology & Electric installation for power supply \\
Air-conditioning technology & Electric installation for illumination \\
Refrigeration technology & Grid surrogate technology \\
Process control & Fire alarm technology \\
Control technology & Shutter and sunblinds technology \\
Sanitary installations & Telephone and Teletype technology \\
Wells and rain water utilization & Sprinkler technology \\
Swimming Pool installation & Person call technology \\
(Drinking-) water preparation & Hoist and lift technology \\
Sewage-, lift station technology & Electric installations \\
Access control, surveillance & Telecommunications \\
Vacuum cleaning installation & \\
\hline \hline
\end{tabular}

Denmark) at the same time for the same application. ISO standardized their field systems several years later in a similar way [17].

Today, many developed fieldbus systems are past; only a few BA standards dominate the markets [18], [19]. It is worth noting that fieldbus systems are now installed in all automation areas. Only, because of political reasons or reasons of competition, or in order to differentiate many companies, standardization organizations and other institutions often name their systems sensor networks and sensor and actuator networks or use similar expressions.

Industry products must be successful in order to make money with them. To convince building owners that they should invest money in fieldbus systems for BA or even SH was very hard. Only a few people saw the advantage. To be able to increase the comfort in a house was not stimulating enough to spend more money than necessary. Only when they could be convinced that energy costs can be reduced and when companies invested in objects like the "Reichstag" in Berlin to show the feasibility that the situation changed completely.

\section{REQUIREMENTS FOR BA}

Table I lists industries which are active in BA [7] (as an example, here, for Austria; similar situations appear in other countries). These industries represent all services that one would expect from BA and SH. It is the art of BA to integrate these historically separate things. The goals are manifold: cost

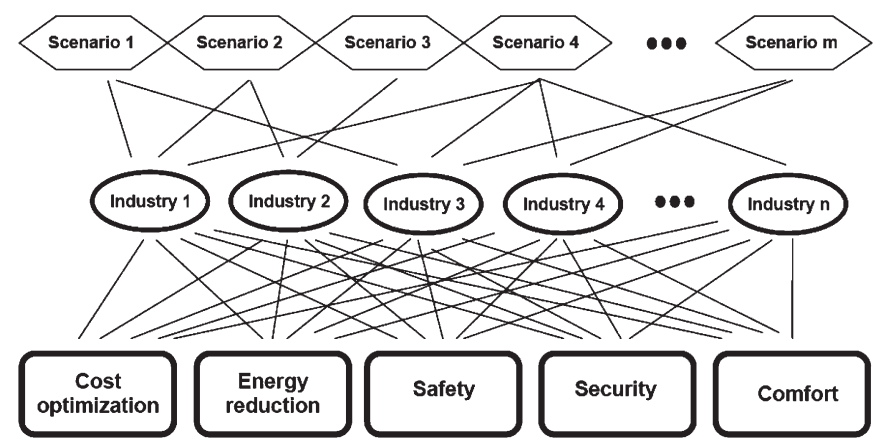

Fig. 4. Scenarios in relation to trades and functions.

optimization, enhanced energy efficiency, increased security, and increased comfort, in summary a multiplication of the functions utilized in a building (Fig. 4).

An example shall illustrate the interdependences: Sunblinds are used in situations when too much sun light or sun heat enters the building. Shading has immediate influence on lighting and heating control by changing both the lighting scenario and the heat radiation in a room. Thus, sunblinds, lighting, and heating are interdependent and should have cooperative control.

Aside from normal operation, interdependences are also given in emergency scenarios, where, depending on the situation, nodes belonging to different industries shall cooperate [e.g., controlling lighting as well as HVAC in the case of fire to ensure person safety]. In situations where the network is affected, it is important to have distributed architectures with nodes that are able to operate even if network connectivity fails.

We see the difference in requirements compared to other fields like automotive or industrial fieldbuses, in which both have a rather limited amount of network nodes usually using the same fieldbus system, while an average building can easily contain thousands of nodes (up to hundred thousands of nodes in large buildings) [20]. The network that is used is provided by a single vendor, and the nodes are not separated into industries but rather grouped by their functionality. This leads to the usage of six or seven layers of the ISO/OSI model in complex BA systems (BASs) in order to manage the complexity of the larger networks. However, the progress showed that even all ISO/OSI layers are not sufficient. Interoperability rules and 
functional profiles as defined by LonMark International [21] were the historic step toward large and complex multivendor installations. Nowadays, these guidelines are often referred to as "8th layer" of the ISO/OSI model. The immense effort put into these definitions shows the importance of these abstract layers compared to almost trivial hardware which does not really matter nowadays in terms of interoperability (the original transport layers are, for instance, more and more replaced by Ethernet). The creation of value in future BA lies in the definition of standards for abstract layers (see also [22]).

However, this is not a scientific challenge. The definition of profiles is a task for the corresponding industry. This includes maintenance and upgrade of already existing profiles. Scientific challenges can be seen in the ever increasing complexity of systems and in the intelligent evaluation of the enormous amounts of collected data [23]-[26]. Another potential for improvement is the interface between fieldbuses, which currently creates unnecessary additional costs. Maybe, it is possible to harmonize them at least in the lower layers ${ }^{4}$ by, e.g., Ethernet [27]-[29]. Maybe, the new developments around OLE for process control and service-oriented architectures can help in providing a solution for the upper layers. More considerations about complexity are detailed in Section V.

Wireless sensor networks (WSNs) are (businesswise) still in their infancy in BA [9]. First of all, their energy consumption, reliability, and security cannot be considered optimal. Additionally, there exist a variety of standards, confusing potential investors. General-purpose WSNs need a transition to BArelevant abilities, which is one point for scientific considerations in the future [30]: Find suitable components which fulfill the requirements, at lowest component costs.

SHs are of large interest for architects, researchers, and persons who simply love modern things. Economically, their network technology plays currently no considerable role. Again, the important factor is costs. The efficiency of planning, implementing, and finally integrating and maintaining is-like in computer engineering - a question of size. A building for one, two, or even three families cannot be equipped with enough components to reach a critical number interesting for individual expert advice. This was the reason for, e.g., KNX [31], to follow a completely different philosophy. Their components are all strongly standardized, low cost, and simplified and can be used (only) for a particular set of scenarios. Flexibility is therefore dramatically reduced, although it is still difficult to find the right experts to integrate a system. In addition, the potential for energy savings is limited, etc. In short, the economic breakthrough is still not there.

\section{Functional SAFETy AND Information SECURITY}

Functional safety, although an important and established topic in transport (avionics, traction, and automotive) or industrial automation (e.g., manufacturing support robots), was, up

\footnotetext{
${ }^{4}$ Harmonizing, for example, the lowest layer will reduce costs by increasing mass production. However, it will not be possible to replace all higher layers and, particularly, the profiles, which were developed by competing companies and user organizations.
}

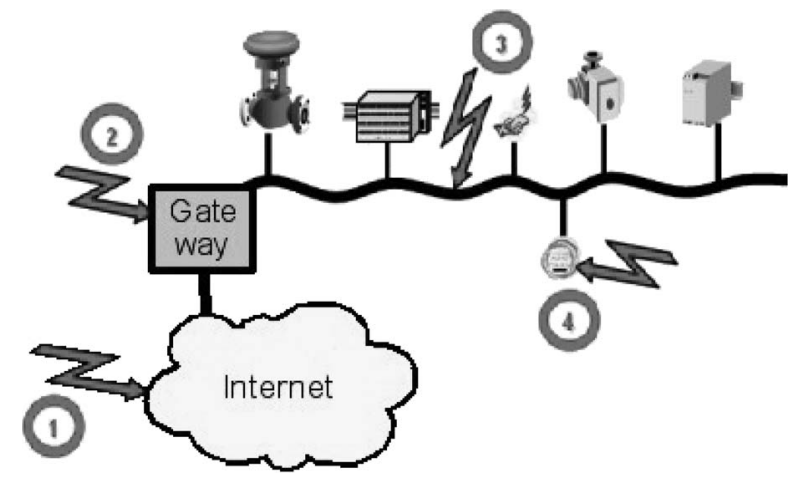

Fig. 5. Potential targets for attacking a BA fieldbus.

to now, not much of an issue in BA. Safety-relevant functions in automated buildings were implemented with stand-alone systems, since the existing BASs were not certified for functional safety. The increased need for unified network management and system integration, on the one hand, and the high costs for safety-certified components lead to a compromise. Safetyrelevant applications (e.g., automated doors, emergency lighting, fire alarm, etc.) and nonsafety-relevant application (HVAC, lighting, etc.) share the same network infrastructure. This leads to easier management and reduced costs of a complex BAS that also satisfies safety requirements, like SafetyLON.

The situation with information security is, in fact, even worse. In the case of safety, buildings were on the "safe side:" If no integrated emergency lighting exists, no one needs safetycertified BA. Security did not have such a safe side. BASs were always security relevant. A classical example is an insider attack of a disappointed employee, using a "Trojan" fieldbus node with a secret wireless channel to outside (see Fig. 5, \#4).

Security features of fieldbuses are currently only very limited or even nonexistent [32]. The compromise has to be found between high interoperability and high security. However, maybe, a solution can be found in scientific investigations with the use of smart cards, as the authors in [32] and [33] already started to think about. The concepts of IT security can certainly be a blueprint for this task. Access control lists, roles, and end-to-end security are state-of-the-art in IT systems. Adding proxy firewalls to fieldbuses is certainly not enough to speak of information security.

\section{ENERGY MANAGEMENT}

One of the most wanted applications of automated buildings and homes is to save operational costs. This is particularly valid for commercial buildings but slowly makes its way into private homes as well. Taking influence on the consumption of buildings and homes falls into the category "demand side management." This ranges from changing light bulbs or insulation up to sophisticated energy information systems (EIS) and automated load management systems. BASs are, however, always a very valuable contribution to energy management.

The possibly most important aspect of energy management is energy efficiency. Efficient buildings can save emissions and costs. The main hurdle for making the right efficiency decisions 
is typically the lack of information. The operators of buildings usually have no idea about where and how their building uses (and maybe wastes) energy and how it compares to similar (size, climate, etc.) buildings. An EIS is the tool of choice for this problem. Integrating a large variety of data sources (sensor networks, meters, databases, statistics, structural data, etc.; see [34]), it can calculate key figures that an energy consultant needs to increase the efficiency of a building.

A second step is active load management. Depending on the incentive, a building might react to online energy prices or to messages from the grid operator. Such demand-responsive buildings are a hot research topic and are expected to be a valuable contribution to stable and economic grid operation [35].

The basis for responsive buildings is the fact that several "processes" in the building allow for temporary shedding. The most prominent ones are those with some hidden storage characteristics or (thermal) inertia like heating or air conditioning. However, even lighting is a candidate for demand response. Dimming the lights by $15 \%$ for an hour will not harm anyone and is fast and clean. ${ }^{5}$

Classically, a building only consumes energy, although that might even change if distributed energy resources and local generation become more widespread. In this case, automation and integration are even more important.

\section{FUtURE Prospects}

As already mentioned earlier, automation faces a complexity problem. For the realization of proper functionality, a sufficiently high number of sensors and actuators are required. These components need to be interconnected and communicate in a reliable, secure, and timely fashion (see approaches in, e.g., [9], [36], and [37]). The amount of data to be transmitted is fortunately quite small-in the case of simple sensors in the range of some bytes every $10 \mathrm{~min}$. On the other hand, more sophisticated sensors as finger print sensors, cameras [38], or the like produce considerably more data [23]-[26]. Altogether, these data soon sum up to considerable amounts and quickly go beyond the capacity of standard computers in the imaginary case of data collection on a single computer. All this information can only be processed in a decentralized manner. Enough processing power and systems that are capable of learning [39] or, at least, adapting capabilities need to be introduced.

Another problem is the commissioning of all the components. The installation is usually performed by cheap semiskilled workers. However, the physical connection between all the nodes is only the basic requirement for proper operation but cannot guarantee the establishment of all communication channels. For the centralized management and maintenance of all data and connections, we are currently lacking the right tools.

Another hot topic of research lies in intelligent sensors, actuators, and devices. The dream of intelligent machines is

\footnotetext{
${ }^{5}$ This is a tradeoff between energy saving and comfort. The advantage of BA is that the tradeoff can be decided on demand.
}

as old as manhood itself [40]. With increasing technological development, the requirements for intelligent devices also increased. However, up to now, artificial intelligence (AI) lacks solutions to the demands of truly intelligent machines that have no problems to integrate themselves into daily human environments. Current hardware with a processing power of billions of operations per second could not substantially contribute to the intelligence of machines compared to early AI times [41]. There are great results, of course. Machines are able to find the shortest path between far apart cities on the map; algorithms let you find information described only by few key words. However, no machine is able to get us a cup of coffee from the kitchen. In addition, machines are not able to recognize situations of humans. Achievements in this direction would allow for automatic surveillance and security systems. Therefore, the system would need the ability to associate unattended luggage in airport environments with a person that may stand some meters away in a queue-or is already on his way out of the building. First products for automatic surveillance are already available to support human security staff, but we can expect much more in this direction in the future also for domestic applications like intelligent environments or ambient assisted living.

The requirements on a metalevel are the same in all applications: perceiving information and interpreting this information with respect to the context of the system. Context is a theoretical construct by humans; it is not contained in the sensor information that is available to a machine. Therefore, for a machine to derive the context, it is necessary to give it the same perceptional function and anticipation as a human-a bionic approach which requires for searching for biologic archetypes. In the case of human's functional capabilities, there are scientific disciplines concerned with it, namely, the humanities. For engineers, humanities have the touch of subjective, maybe imprecise, sciences. The latter is simply an illegitimate prejudice by people not from the field, whereas the first impression is a consequence of the matter with which many fields in humanities are concerned: the subjective self [42]. A technical system with humanlike capabilities needs to possess a combination of predefined "mental content" in the presented structure [41] and learning capabilities [39] for its particular application area. Current approaches focus on fault detection [43], [44] using statistical methods [45]. Research in this direction is still in its infancy; however, it will change the impact of automation in our daily life widely.

Another also very important topic in all fields of automation is sensor technology. Sensors need to become smaller and cheaper, on the one hand, and they need to perceive more aspects of the environment and preprocess the data, on the other hand. Nanotechnology already provides us with new powerful devices like laboratory-on-chip devices or detectors of all kinds of molecules in the air. It can be anticipated that sensor technology in the future is able to more and more deliver all kinds of required information for monitoring all kinds of processes within a building (speaking of building-relevant or user-relevant processes, not, e.g., manufacturing processes in industrial buildings). 
Another upcoming challenge is the design of BASs for large buildings, which contains a considerable amount of repetitive tasks that have some need for automation themselves (e.g., designing HVAC for all rooms in an office building). A solution is to use design patterns, which are based on standardized hardware modules. However, this will only work properly (i.e., yield an interoperable system) if the whole process starting from selecting modules, evaluating interoperability, and system composition is thought through well [46], and communication issues are considered as well [47].

Embedded systems will allow integrating many of these sensors, together with a preprocessing unit and wired and wireless communication possibilities, into single-chip solutions affordable for a broad segment of customers.

Today's buildings are at a technological stage of a Volkswagen Beetle (a very popular small car in central Europe and Mexico that was designed in the 1960s) but will be equipped with modern technology soon-as what happened in many other fields of automation before.

\section{REFERENCES}

[1] Webster's—New Encyclopedic Dictionary, Könemann, Cologne, Germany, 1994. Library of Congress Cataloging in Publication.

[2] Brockhaus Enzyklopädie in 24 Bänden, 19th ed., F. A. Brockhaus, Mannheim, Germany, 1989.

[3] Building Automation in Wikipedia. [Online]. Available: http://en. wikipedia.org/wiki/Buil-ding_automation

[4] Building Automation (German article) in Wikipedia. [Online]. Available: http://de.wikipedia.org/wiki/Gebäudeautomatisierung

[5] D. Loy, D. Dietrich, and H.-J. Schweinzer, OpenControl NetworksLonWorks/EIA 709 Technology. Norwell, MA: Kluwer, 2001.

[6] IES Technical Committee on Building Automation, Control and Management, [Online]. Available: http://ieee-ies.org/tc/bacm/

[7] K. Kabitzsch, D. Dietrich, and G. Pratl, Eds., LonWorks Gewerkeübergreifende Systeme. Düsseldorf, Germany: VDE Verlag, 2002.

[8] D. Dietrich and P. Fischer, LonWorks-Planerhandbuch. Düsseldorf, Germany: VDE Verlag, 2001.

[9] D. Egan, "The emergence of ZigBee in building automation and industrial control," Comput. Control Eng. J., vol. 16, no. 2, pp. 14-19, Apr. 2005.

[10] S. T. Bushby, "BACnet: A standard communication infrastructure for intelligent buildings," Autom. Construction, vol. 6, no. 5/6, pp. 529-540, Sep. 1997.

[11] J. Ploennigs, M. Neugebauer, and K. Kabitzsch, "A traffic model for networked devices in the building automation," in Proc. WFCS, 2004, pp. 137-145.

[12] M. Neugebauer, J. Plönnigs, and K. Kabitzsch, "Prediction of network load in building automation," in Proc. IFAC FET, 2003, pp. 269-274.

[13] W. Granzer, W. Kastner, G. Neugschwandtner, and F. Praus, "Security in networked building automation systems," in Proc. WFCS, 2006, pp. 283-292.

[14] 8th IFAC International Conference on Fieldbuses \& Networks in Industrial \& Embedded Systems, [Online]. Available: http://www.fet2009.org/

[15] 7th IEEE International Workshop on Factory Communication Systems, [Online]. Available: http://iai82110.inf.tu-dresden.de/wfcs2008/

[16] D. Dietrich, "Evolution potentials for fieldbus systems," in Proc. 3rd IEEE WFCS, Porto, Portugal, 2000, pp. 145-146.

[17] T. Sauter, "Fieldbus Systems-Embedded Networks for Automation," in Networked Embedded Systems Handbook, R. Zurawski, Ed. Boca Raton, FL: CRC, 2009, pp. 20-1-20-64.

[18] W. Kastner, G. Neugschwandtner, S. Soucek, and H. M. Newman, "Communication systems for building automation and control," Proc. IEEE, vol. 93, no. 6, pp. 1178-1203, Jun. 2005.

[19] D. Snoonian, "Smart building," IEEE Spectrum, vol. 40, no. 8, pp. 18-23, Aug. 2003.

[20] A. W. Colombo, R. Schoop, and R. Neubert, "An agent-based intelligent control platform for industrial holonic manufacturing systems," IEEE Trans. Ind. Electron., vol. 53, no. 1, pp. 322-337, Feb. 2006.

[21] LonMark International, [Online]. Available: http://www.lonmark.org/
[22] M. Wollschlaeger, H. Kulzer, D. Nuebling, and P. Wenzel, "A common model for XML descriptions in automation," in Proc. IFAC World Congr. Prague, Czech Republic, 2005.

[23] C.-H. Lu and C.-C. Tsai, "Adaptive predictive control with recurrent neural network for industrial processes: An application to temperature control of a variable-frequency oil-cooling machine," IEEE Trans. Ind. Electron., vol. 55, no. 3, pp. 1366-1375, Mar. 2008.

[24] C. Guo, Q. Song, and W. Cai, "A neural network assisted cascade control system for air handling unit," IEEE Trans. Ind. Electron., vol. 54, no. 1, pp. 620-628, Feb. 2007.

[25] H. K. Lam and F. H. F. Leung, "Design and training for combinational neural-logic systems," IEEE Trans. Ind. Electron., vol. 54, no. 1, pp. 612 619, Feb. 2007.

[26] C.-F. Juang, C.-M. Lu, C. Lo, and C.-Y. Wang, "Ant colony optimization algorithm for fuzzy controller design and its FPGA implementation," IEEE Trans. Ind. Electron., vol. 55, no. 3, pp. 1453-1462, Mar. 2008.

[27] M. Felser and T. Sauter, "Standardization of industrial ethernet-The next battlefield?" in Proc. IEEE Workshop Factory Commun. Syst., Wien, Austria, Sep. 22-24, 2004, pp. 413-421.

[28] M. Lobashov and T. Sauter, "Vertical communication from the enterprise level to the factory floor-Integrating fieldbus and IP-based networks," in Proc. 11th IEEE Emerg. Technol. Factory Automat., Praha, Czech Republic, Sep. 20-22, 2006, pp. 1214-1221.

[29] J.-D. Decotignie, "The many faces of industrial ethernet," IEEE Ind. Electron. Mag., vol. 3, no. 1, pp. 8-19, Mar. 2009.

[30] J. Glaser, D. Weber, S. Madani, and S. Mahlknecht, "Power aware simulation framework for wireless sensor networks and nodes," EURASIP J. Embedded Syst., vol. 2008, pp. 1-16, 2008.

[31] KNX. [Online]. Available: http://www.knx.org/

[32] D. Dietrich, P. Palensky, and A. Treytl, "Communication in automation with the emphasis on security," in Proc. Int. Workshop Integr. Syst. Focus Fire Security, Rome, Italy, 2003, vol. 17, pp. 89-104.

[33] P. Palensky, "Smart card security for field area networks," in Proc. IEEE-SIBCON, 2003, pp. 135-138.

[34] P. Palensky, "The JEVis service platform—Distributed energy data acquisition and management," in The Industrial Information Technology Handbook, R. Zurawski, Ed. Boca Raton, FL: CRC, 2005, pp. 111-121.

[35] M. A. Piette, D. Watson, N. Motegi, S. Kiliccote, and E. Linkugel, "Participation through automation: Fully automated critical peak pricing in commercial buildings," in Proc. ACEEE Summer Study Energy Efficiency Buildings, Pacific Grove, CA, Aug. 13-18, 2006.

[36] T. Li and Y. Fujimoto, "Control system with high-speed and realtime communication links," IEEE Trans. Ind. Electron., vol. 55, no. 4, pp. 1548-1557, Apr. 2008.

[37] M. H. Shwehdi and A. Z. Khan, "A power line data communication interface using spread spectrum technology in home automation," IEEE Trans. Power Del., vol. 11, no. 3, pp. 1232-1237, Jul. 1996.

[38] T. Bucher, C. Curio, J. Edelbrunner, C. Igel, D. Kastrup, I. Leefken, G. Lorenz, A. Steinhage, and W. von Seelen, "Image processing and behavior planning for intelligent vehicles," IEEE Trans. Ind. Electron., vol. 50, no. 1, pp. 62-75, Feb. 2003.

[39] D. Bruckner, Probabilistic Models in Building Automation-Recognizing Scenarios With Statistical Methods. Saarbrücken, Germany: VDMVerlag Dr. Müller, 2008.

[40] P. Wide, "The electronic head: A virtual quality instrument," IEEE Trans. Ind. Electron., vol. 48, no. 4, pp. 766-769, Aug. 2001.

[41] D. Dietrich, G. Fodor, G. Zucker, and D. Bruckner, Eds., Simulating the Mind-A Technical Neuropsychoanalytical Approach. New York: Springer-Verlag, 2008.

[42] M. Solms and O. Turnbull, The Brain and the Inner World. New York: LLC, 2002

[43] J. Schein and J. M. House, "Application of control charts for detecting faults in variable-air-volume boxes," ASHRAE Trans., vol. 109, pp. 671682, 2003.

[44] J. M. House, W. Y. Lee, and D. R. Shin, "Classification techniques for fault detection and diagnosis of an air-handling unit," ASHRAE Trans., vol. 105, no. 1, pp. 1987-1997, 1999.

[45] P. R. Goulding, B. Lennox, D. J. Sandoz, K. Smith, and .O Marjanovic, "Fault detection in continuous processes using multivariate statistical methods," Int. J. Syst. Sci., vol. 31, no. 11, pp. 1459-1471, Nov. 2000.

[46] C. Hahn, A. Gossling, R. Frenzel, and M. Wollschlaeger, "Towards an automated generation of device firmware components for intelligent field devices," in Proc. ICIT, 2009, pp. 1-6.

[47] J. Ploennings, M. Guertler, M. Neugebauer, and K. Kabitzsch, "Automated measurement-based device traffic modelling in control networks," in Proc. 5th IEEE INDIN, 2007, pp. 27-32. 


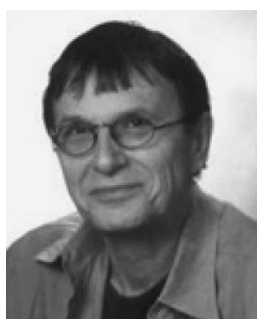

Dietmar Dietrich (SM'04) received the M.Sc. degree in Karlsruhe, Germany, in 1977 and the Ph.D. degree in Berlin, Germany, in 1981.

Since 1992, he has been a Professor of computer technology with the Institute of Computer Technology, Vienna University of Technology, Vienna, Austria, where he was the Head of the institute from 1999 until 2008. Prior to this, he worked in the aviation and space industry and later in the communication industry. His research interests were in digital application-specified integrated circuit design and, currently, are mainly in communication systems - particularly on fieldbus systems - as well as in modeling of psychoanalytical mental apparatus for developing intelligent automation systems.

Mr. Dietrich is a member (member of the advisory board, Chair, convenor, etc.) of various national and international organizations and also a delegate of the Austrian Electrotechnical Association (OVE) and Austrian Standards Institute (ON) in the European Committee for Standardization (CEN) and the European Committee for Electrotechnical Standardization (CENELEC). He has been an Associate Editor of the IEEE TRANSACTIONS ON INDUSTRIAL INFORMATICS and is an Associate Editor of the IEEE TRANSACTIONS ON INDUSTRIAL ELECTRONICS. He is an ADCOM member, initiator, and past Technical Committee Chair of the Building Automation, Control and Management in IES/IEEE. Furthermore, he is a Coorganizer of various tracks and conferences for IEEE. He also became a member of the board of the OVE in 2002 and then was Vice President until spring 2008. He was the Chair of Section Austria in IEEE R8 from 2006 to 2008. He founded the Center of Excellence for Fieldbus Systems in 1994 and the international biennial fieldbus conference $\mathrm{FeT}$ in 1995. He has organized national and international workshops and conferences of IEEE and International Federation of Automatic Control. He is an Associate Editor for the European Association for Signal Processing.

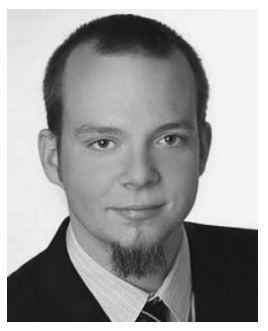

Dietmar Bruckner (M'05-SM'10) received the M.Sc. degree in electrical engineering and information technology and the Ph.D. degree in technical sciences from Vienna University of Technology, Vienna, Austria, in 2004 and 2007, respectively.

$\mathrm{He}$ has been with Vienna University of Technology since 2004, where he was first a Project Assistant and has been an Assistant Professor since May 2009. He has been managing the cognitive automation research group since 2007. His research interests include ambient assisted living, intelligent environments, intelligent automation systems, and, in general, models for more intelligent decision units.

Dr. Bruckner is a member of the IEEE Industrial Electronics Society (IES) and the Austrian Electrotechnical Association (OVE). He holds the positions as Secretary of the IES Technical Committee on Building Automation, Control and Management and as Coordinator of the IEEE Section Austria Chapter.

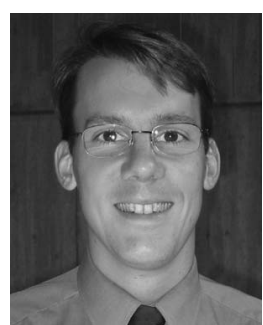

Gerhard Zucker (M'05) received the M.Sc. degree in electrical engineering and information technology and the Ph.D. degree in technical sciences, a vivavoce exam with excellence, from Vienna University of Technology, Vienna, Austria, in 1998 and 2006, respectively.

$\mathrm{He}$ is currently a Senior Researcher with the Austrian Institute of Technology, Vienna, working in the field of sustainable building technology. He worked on different projects in the field of building automation and artificial intelligence before he began the technical project management of various projects on basic research as well as projects in cooperation with different companies. He is the Editor of two books and numerous scientific publications. His research interests include building automation and controls for optimization of energy efficiency. $\mathrm{He}$ specializes in methods to recognize and evaluate behavior of persons for optimizing building usage and maximizing comfort.

Dr. Zucker is a member of the IEEE Industrial Electronics Society Technical Committee on Building Automation, Control, and Management. He has been a Session Chair and Track Chair for various conferences, the latest being Track Chair for the IEEE Conference on Industrial Informatics 2010 and Special Session Chair for the IEEE Human System Interaction 2010.

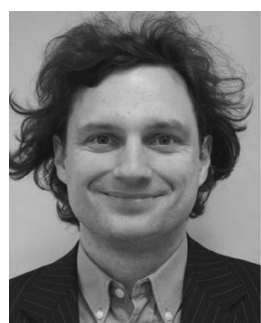

Peter Palensky (SM'05) received the Ph.D. degree from Vienna University of Technology, Vienna, Austria, in 2001.

$\mathrm{He}$ is currently the Head of the business unit for "Sustainable Building Technologies," Austrian Institute of Technology, Vienna. His research field is in distributed systems and their application for automation, particularly energy and building automation.

Dr. Palensky is active in many international committees like the International Organization for Standardization or CEN. He is an IEEE IES ADCOM member and Chair of its Technical Committee on Building Automation, Control and Management. 